\title{
A Knowledge Integration Methodology for Developing Customized Maintenance Documents
}

\author{
Ying Huang \\ Department of Mechanical \\ Engineering, \\ Taizhou University, \\ Taizhou 318000, China
}

samanthanew@gmail.com

\author{
Mickaël Gardoni \\ Department of Automated \\ Production, ETS Montréal, \\ Montréal, Québec, Canada \\ mickael.gardoni@etsmtl.ca
}

\author{
Xingjun Wang \\ Department of Physics and \\ Electronic Science, \\ Qiannan Normal College for \\ Nationalities, \\ Duyun 558000, China \\ xingjun.wang@qq.com \\ Coulibaly Amadou \\ LGECO, INSA de Strasbourg, \\ Strasbourg Cedex, France \\ amadou.coulibaly@insa- \\ strasbourg.fr
}

\begin{abstract}
This paper presents a knowledge integration methodology for developing Customized Maintenance Document (CMD) systems. They are adaptive and interactive maintenance support systems that employ product information, expert knowledge, and hypermedia to provide correct support in the process of performing the maintenance task. The methodology emphasizes requirements of CMDs, information organization, utilization of expert knowledge, and the mechanisms for customizing maintenance tasks. The methodology shows how knowledge about utilization conditions, environments, and maintenance constraints can be integrated and used to deliver personalized and task-oriented maintenance support. The overall framework and approaches have been implemented and applied to a transportation enterprise with four heavy-duty trucks as an exemplary case of demo in principle.
\end{abstract}

Keywords: Customized Maintenance Document, knowledge-based systems, Integration of knowledge, personalized support, user model

Material published as part of this publication, either on-line or in print, is copyrighted by the Informing Science Institute. Permission to make digital or paper copy of part or all of these works for personal or classroom use is granted without fee provided that the copies are not made or distributed for profit or commercial advantage AND that copies 1) bear this notice in full and 2) give the full citation on the first page. It is permissible to abstract these works so long as credit is given. To copy in all other cases or to republish or to post on a server or to redistribute to lists requires specific permission and payment of a fee. Contact Publisher@,InformingScience.org to request redistribution permission.

\section{Introduction}

As the market competition becomes more drastic, the traditional sellers' market has been transformed to a buyers' market in today's industries. More and more manufacturers realize the importance of the product manual, as customers not only require quality product, but also focus on product delivery, cost 
of product, and after service (Xia, Xi, Lee, \& Zhou, 2011). Users often form an opinion about a product and its manufacturer and supplier based on their experience of learning how to use and maintain the product.

These and other studies motivated active research during the 1990s that led to transforming the whole area of product documentation. Paper-based technical manuals were replaced by electronic systems which incorporated multimedia and hypermedia elements. The traditional maintenance document is difficult to update in order to follow different utilization conditions. Therefore, the end user or technician needs more time to decide when and what appropriate maintenance task should be accomplished. On the other hand, users often need information in a technical manual to supplement his or her knowledge and experience with the equipment. Required information to accomplish a task may be scattered in different documents. For complex systems like automobiles, airplanes, and machine tools, most of these documents consist of a huge amount of documentation containing disassembling/assembling instructions and drawings of parts, subassemblies, and exploded views. It is difficult for users to find a piece of information they need amongst this huge amount of documentation. Then, how to organize the supporting information in a right form in order to facilitate the user performance is another problem which should be highlighted.

The goal of this article is to outline a methodology for elaborating Customized Maintenance Documents (CMDs), which shows how to develop the document in line with (1) various maintenance strategies triggered by variety of utilization conditions and (2) various maintenance program schedule caused by constraints, such as available tools and materials, maintenance environment, and cost. This paper also emphasizes how to supply personalized maintenance support for users with different backgrounds in terms of skill levels, experience, favorites, etc. Then, in order to elaborate the document adaptively according to the above factors, some enabled method and mechanism are discussed in this paper, such as inference mechanism, information organization (Brodie, Gupta, \& Shi, 2005), and design of knowledge base (Batanov, Nagarur, \& Nitikhunkasem, 1993).

The rest of this paper is organized as follows. The next section briefly introduces related works about the development of product manuals. The third section presents the methodology for developing CMDs by describing the main requirements, knowledge management, and framework of CMDs. The proposed methodology through an exemplary case is demonstrated in the fourth section. Finally, the paper concludes with discussion of further research directions.

\section{Related Works}

Many researchers devote themselves to the problem of developing product manuals ranging from the Interactive Electronic Technical Manual (IETM) to the more advanced Intelligent Product Manual (IPM). The CD-based and stand-alone applications have been transformed into distributed web-based product support systems. Also, hypermedia, concurrent engineering, and knowledge-based approaches are used in this development. Generally, there are two research streams: some research teams emphasize the provision of different levels of information to different groups of users, and others emphasize the supporting maintenance work such as diagnosis and repair of failures.

A typical representative of the first group is IPM, which is an interactive product support system providing just-in-time support to the user during the life of the product. Case-Based Reasoning (CBR) was employed (Pham, Dimov, \& Soroka, 2001) for providing the different levels of detail and presentation format of information for the different categories of users (who have different levels of expertise, qualification, and experience). R.M. Setchi and his associates (Setchi, Pham, \& Dimov, 2006) tried to structure the manual to ensure the provision of different levels of infor- 
mation to particular groups of users and their tasks. They were also interested in knowledge acquisition (Pham, \& Setchi, 2001), data and information models (Pham, Dimov, \& Huneiti, 2003), and product manual modeling (Setchi, Lagos, \& Huneiti, 2007).

IETM is the original prototype of the second group. Initially, IETM emerged to solve the problems inherent in paper-based product documents such as paper cost, weight, volume, printing, and deterioration. In order to make it more useful, especially in the maintenance phase, Cooper (1999) proposed an adaptive diagnostics and personalized technical support system (ADAPTS) at the base of IETM. The system accepts real-time inputs from Condition-Based Maintenance (CBM) systems and then plans a course of action for diagnosing and repairing equipment failure. Nowadays, the US Navy and Army utilize an integrated product support system for condition-based monitoring of helicopters (Mathur, Ghoshal, Haste, Domagala, Shrestha, Malepati, \& Pattipati, 2000). There are many similar systems such as the 'Virtual Maintenance System' (Van Houten, \& Kimura, 2000) and Intelligent Maintenance System (IMS) (Lee, Ghaffari, \& Elmellgy, 2011).

From the literature reviewed above, it becomes clear that there are proven methodologies such as IETM for designing product support solutions for today's manufacturing products in the level of information, but they did not focus on the maintenance level. Even though some of them integrate the Condition-Based Maintenance system with product manuals, they just emphasize on predicting possible failures or future states of a mechanical system. Finally, most of them degenerated into an information research tool. However, there are almost no product manuals that deliver customized maintenance support with consideration of maintenance strategy, maintenance programme planning, user background, etc.

\section{The CMD Methodology}

\section{Requirements of CMDs}

The main assumption underlying this work is that CMDs can provide adaptive and personalized maintenance support and overcome the basic deficiencies of conventional maintenance manuals on their structure, content and form, and the traditional techniques. Function needs of CMDs are discussed from three aspects separately: data requirements, maintenance support requirements, and requirements of presentation mechanism.

\section{Data requirements of CMDs}

One of the most significant targets of CMDs is the transition from an intensive, non-integrated environment to a highly integrated product maintenance and support environment. To achieve this goal and optimize the authoring process of maintenance manuals, CMDs development is closely associated and directly integrated with the product data environment. Two main types of data are considered in this research: product data, and technical data in the forms of engineering data, schematics, drawings, product models, assembly tress, bills-of-materials, and technical manuals.

Product data is considered because it is one of the essential and most data-intensive elements of the logistics portion in the digital environment. Product data is the technical and management data required to field, operate, and sustain mechanical systems. Product data users can broaden the definition of product data (e.g., provisioning technical documentation, support equipment recommendation data, engineering change proposals, etc.) to include all the data required to support a mechanical system.

Technical data includes all the necessary information such as technical documents, standards and requirements which guide the technician through an operating procedure or maintenance procedure. These standards are designed to control unexpected release of hazardous energy or startup 
of machines or equipment, and to prevent injury to employees performing servicing or maintenance. These data objects may be a complete logical data unit, such as a technical specification, an instruction manual, or a maintenance manual. They also may be some document fragments, for example, a part description of a product feature which is more independent and complete logically.

\section{Requirements of maintenance support}

The identification of an effective maintenance programme is a critical issue for mechanical products as it directly affects the operational regularity and capability of the equipment to meet the demands as planned. Therefore, a good maintenance document should provide certain strategic supports in order to perform preventive maintenance actions which are absolutely necessary and cost-effective.

In order to keep maintenance time at a minimum, generally tasks can be grouped together rather than being completed one at a time as they come due. Tasks can be grouped by whether a heavy maintenance environment is needed or not. For example, one group of checks occurs most frequently and has the fewest number of tasks. Typically, such checks can be performed overnight in a line maintenance environment. In contrast, another group of checks comprises a greater number of tasks, many of which require a substantial amount of time to complete. These checks are typically performed in a heavy maintenance environment.

Also, a different logic formula should be selected to determine the time limitation of each task. With one logic formula, parts are changed at standard times regardless of whether the part actually needs to be changed. With another logic formula, parts are not replaced until broken, which allows the time limitation to be revised based upon reliability data for the part during its life cycle. This issue involves the problem of maintenance programme planning.

\section{Requirements of presentation mechanism}

An important aim of CMDs is to enable users to improve their performance by providing them with information that is closest to their current needs. Instead of the hierarchical structure, CMDs take the hypermedia structure which permits the user to move easily between different items of information in a structured fashion.

Hypermedia capabilities include associative linking of information items, management of documents within large hyper-bases, easy navigation, and efficient information retrieval. These hypermedia capabilities facilitate task-oriented structuring of information sources in CMD and extraction of specific information. In addition to traditional multimedia facilities (text, sound, video, and 2D animation), 3D CAD product data are used to create Virtual Reality scenes and 3D animations. The interactive visual presentation of complex procedures by using animations, video clips, virtual reality technology, and expert system modules can provide the maintainer with improved comprehension of technical information. The benefits of audio, in addition to visual notification windows, can improve the delivery of WARNINGs, CAUTIONs, and NOTEs in troubleshooting and maintenance procedures.

\section{Task Recommendation Method}

In this research, we focus on customizing maintenance tasks. The maintenance requirement of mechanical products often varies since these products operate on different levels of stress or load in the real operational environment. Therefore, the failure rate prediction for each component is very complex and extremely difficult to model. In most cases, first, it is nearly impossible to establish precise and accurate models for these systems; second, even if they can be modeled, it is very expensive to produce a solution in real time. Instead of solving the problem in real time each 
time it occurs, an alternative approach is to solve the problem once and memorize the solution for later applying to similar problems. Thus, Case-Based Reasoning is adopted as an ideal method for linking together the different data sources and re-using previous experiences in the current context to solve new problems.

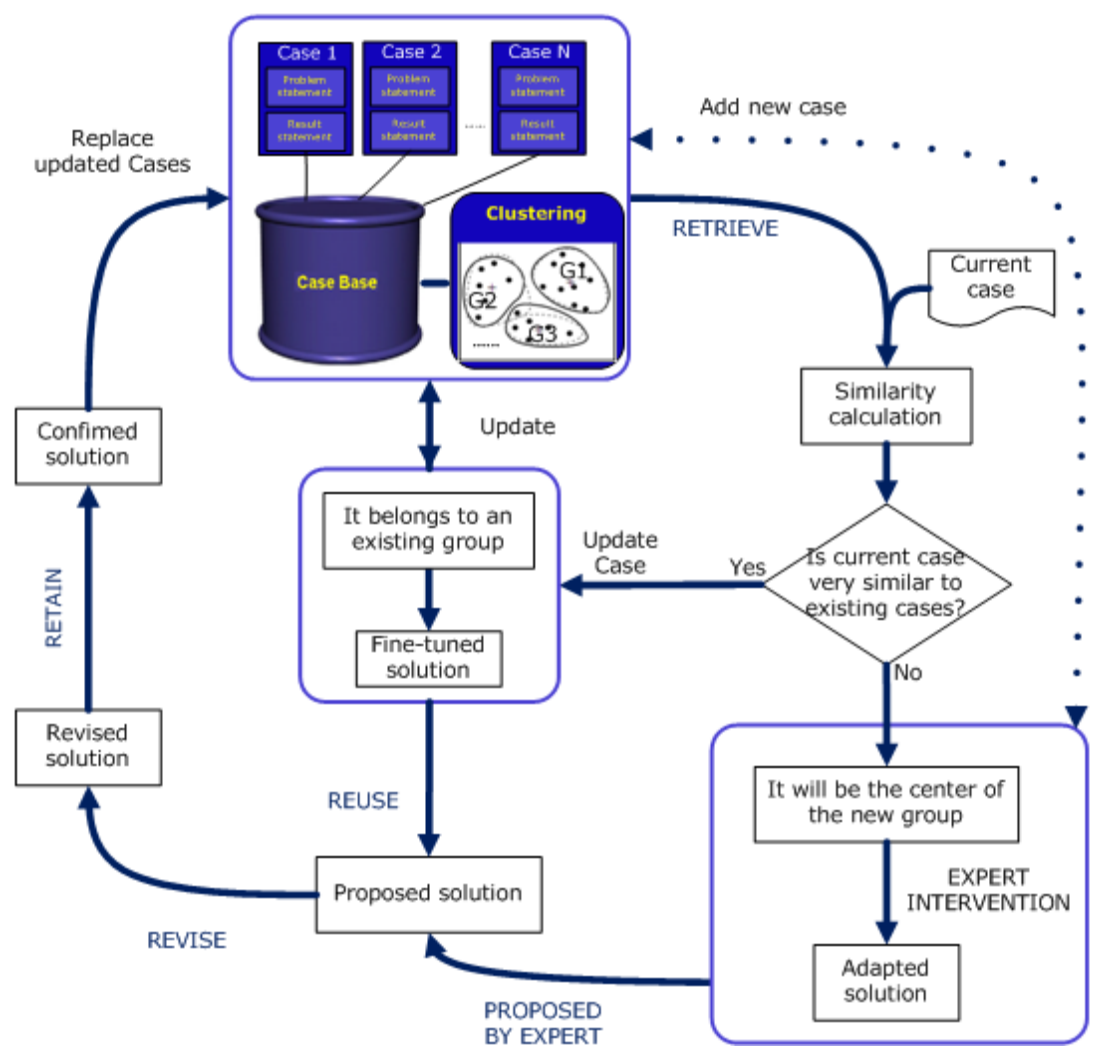

Figure 1. Task recommendation method

As shown in Figure 1, four steps are involved in the task recommendation method.

1) In the first step, a case base is developed for the CMD system to provide a baseline for the application during the initialization phase.

2) In the second step (case retrieval), instead of retrieving the most similar cases directly, initial cases are clustered into groups based on calculation of between-case similarities, for efficiency of case matching.

3) In the third step (case re-use), if the most similar case is found, then the new case is assigned to an exiting group, and task requirements of other cases in the group can be recommended as a fine-tuned solution.

4) Otherwise, intervention by domain experts is needed since no case can be re-used. In addition, the current case should be added into the case base as a new case.

\section{Knowledge Management}

Maintenance documents contain a large amount of information that can be obtained from a number of sources: paper-based conventional manuals, market surveys, drawings, customer support information, etc. All forms of data are divided into two groups: one is textual data (includes video, graphics, drawing and 3D model), and the other is non-textual data which can be marked up 
by XML. CMDs have to provide efficient access to the stored information and facilitate its application in deriving and formulating answers to questions from the user. Knowledge modeling and knowledge base are two critical parts of knowledge management.

\section{Knowledge modeling}

In order to best extract meaningful knowledge, a semantic data model for CMDs is proposed based on an information usage analysis. This analysis aims at abstracting the intended purpose for the product information, the supported user tasks, and the functional characteristics of the product information elements. These abstract views are combined together into a task-oriented semantic network and set of rules and constraints. The proposed semantic model consists of three layers, Data Layer, Task Layer, and Structure Layer, as shown in Figure 2.

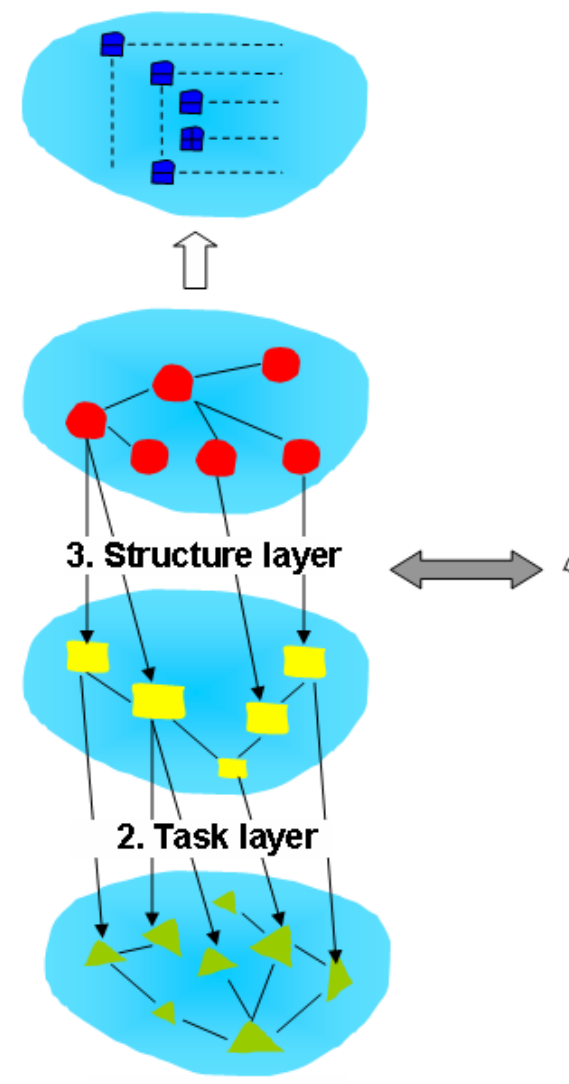

1. Data layer

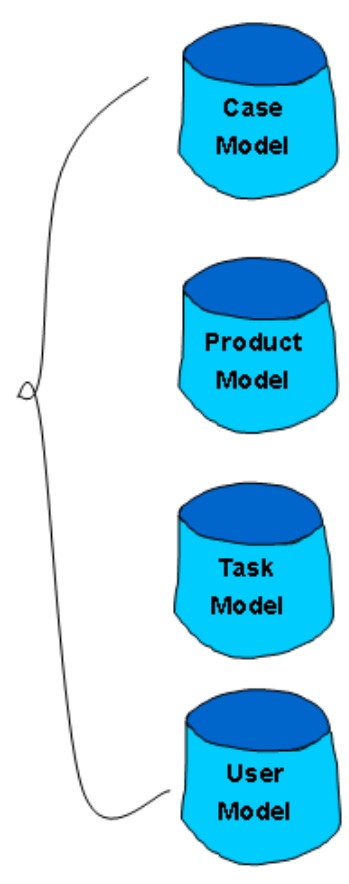

Knowledge Base

Figure 2. Information extraction modeling and enabled engines

The first layer is the Data Layer - a data source describing layer, to be exact - using the "data module" concept which is defined as the smallest self-contained information unit within a technical publication. S1000D, an international specification of technical publication, has been adopted in our study because the same data module can be re-used and re-distributed in many other different projects or publications. This can lead to a tremendous saving of time in the authoring process. Furthermore, the maintenance documents assembled by data modules can be easily maintained and updated, since only the corresponding modules need to be modified for new products or applications. 
The second layer, Task Layer, is a task-oriented layer of document elements which collects information objects together in support of a product version for different applications. The document element does not produce output. It is a staging area that creates an inventory of data modules intended for a particular information fragment. Then, a document fragment identifies the data modules required for a particular output instance. A discreet piece of information is a thematically related unit of content mapped to a particular task (e.g., changing oil in an engine, a list of materials associated with an operation, or a test procedure for surface cracks using magnetic materials).

The third layer, Structure Layer, is another view of information description in order to meet the maintenance support and personalized requirements. On one hand, a relative maintenance task is chosen in line with the maintenance strategy, and its performance sequences are decided by an optimization method. On the other hand, the information is organized in such a way that ensures its homogeneity, validity, and effectiveness of task supporting, according to the user's special interests, experience, and conditions.

\section{Knowledge base}

Unlike static data in a database, the information combined in a CMD has to be of an active nature. That is, it should be employable for generating new information dynamically through processes of logical deduction, environment analysis, or analogical reasoning. Therefore, knowledge -more than just information -- is required for CMDs.

Here, four models are used to create a knowledge base in which detailed characteristics of the CMDs are collected and stored in a database. These are: user model, task model, product model, and case model, as shown in Figure 2.

\section{- User model}

End-users have different levels of expertise, qualification, experience, and responsibility. There are three levels of user: end user, technician (middle level), and manufacturer. The analysis focuses primarily on the background information and interests of a person. The element $<$ Background $>$ contains information about the effective factors of the maintenance task such as usage profile, user profile, skill level, and experience. The element $<$ Interest $>$ is used to contain information about the person's preference such as frequency of searching for certain information fragments, satisfaction feedback, and preference of display type. The difficult question is how to assess the user's knowledge in certain aspects of the domain area. A possible solution could be to interview the user each time he uses the system and to rely on the user's personal judgment of his knowledge about the tasks.

\section{- Task model}

Task is a goal to achieve, which involves a determined change of an object's state (Bertolini, \& Bevilacqua, 2006). In our research, it is assumed that the aim of using a CMD is to perform a set of preventive maintenance (PM) tasks and a set of corrective maintenance (CM) tasks, such as lubrication/servicing, operational/visual, inspection/functional, restoration, and discard (Huang, Gardoni, \& Coulibaly, 2009). The knowledge about the maintenance tasks is represented in a form of task model based on the task taxonomy. Tasks are represented in a hierarchical form and mapped to user profiles. In most cases, the task taxonomy is relatively well standardized and a task catalogue can be used to structure task knowledge. As the time for searching and reading/learning has to be as short as possible, the tasks have to be further differentiated in order to facilitate retrieval and assimilation of knowledge. 


\section{- Product model}

The model includes data created during product development as well as data generated especially for the purpose of developing a product manual. Normally, the structure of a product model follows the topology of the product (product, systems, subsystems, components, etc.). A component can be decomposed into subcomponents; for example, engine as a component can be decomposed into inlet, compressor, combustor, turbine, nozzle, etc., and in turn the subcomponents can be decomposed further; For example, the turbine as a component can be decomposed into subcomponents such as stator and rotator.

\section{- Case model}

Cases can be represented in a variety of forms using the full range of AI representational formalisms including frames, objects, predicates, semantic networks, and rules. The choice of a particular formalism is largely governed by the case content. The collected data are organized as a "cause-result" pair which includes two parts: the first, a set of objective attributes which may contribute to the failure of the mechanical product; and the second, a set of failure attributes describing the health condition of the components which we are interested in. In general, objective attributes do not tend to be very complex, consisting largely of descriptive adjectives, nouns, or values.

\section{Framework of CMDs}

The key objective of this framework is to enable and promote an adaptive and personalized approach to deliver appropriate information for the end user and technician during maintenance sequence. In order to support the adaptive and cost-effective maintenance strategy, expert knowledge and historical information is required to help a technician to make the right decision on what-to-do. Therefore, a more appropriate way of providing recommendation services is to create an interactive environment in which the technician can describe to the CMD system his typical Utilization Conditions. Then, the system can use the knowledge from previous technicians together with built-in domain knowledge to find an ideal maintenance strategy for the technician. This is similar to the scenario where the technician communicates with a human expert who can provide certain knowledge about the reliability of mechanical products and professional experience in this domain, and asks for suggestions in decision making. To provide such services, we need a system that exploits expert knowledge and information of Utilization Conditions to generate recommendations of e-maintenance documents. This section describes how we designed and implemented such a system.

In this work a method of multiple collaborative engines is adopted. Each engine performs a specific task, and different engines work simultaneously to achieve the overall task. To achieve the above goal, our system mainly includes four engines: Knowledge Engine, Diagnostic Engine, Customizing Engine, and Hypermedia Engine. The overall system framework is illustrated in Figure 3 and described below.

1. Knowledge Engine decides which maintenance strategy should be carried out to achieve the desired reliability. Characteristics of the environment are collected from the technician through a list of qualitative questions. The characteristics of the product collected by users should be generalized and normalized in order to measure the similarity between the current case and previous cases. Domain experts employ the multi-attribute method to retrieve the most similar case or cases, and update the Case Base with the confirmed solution. 


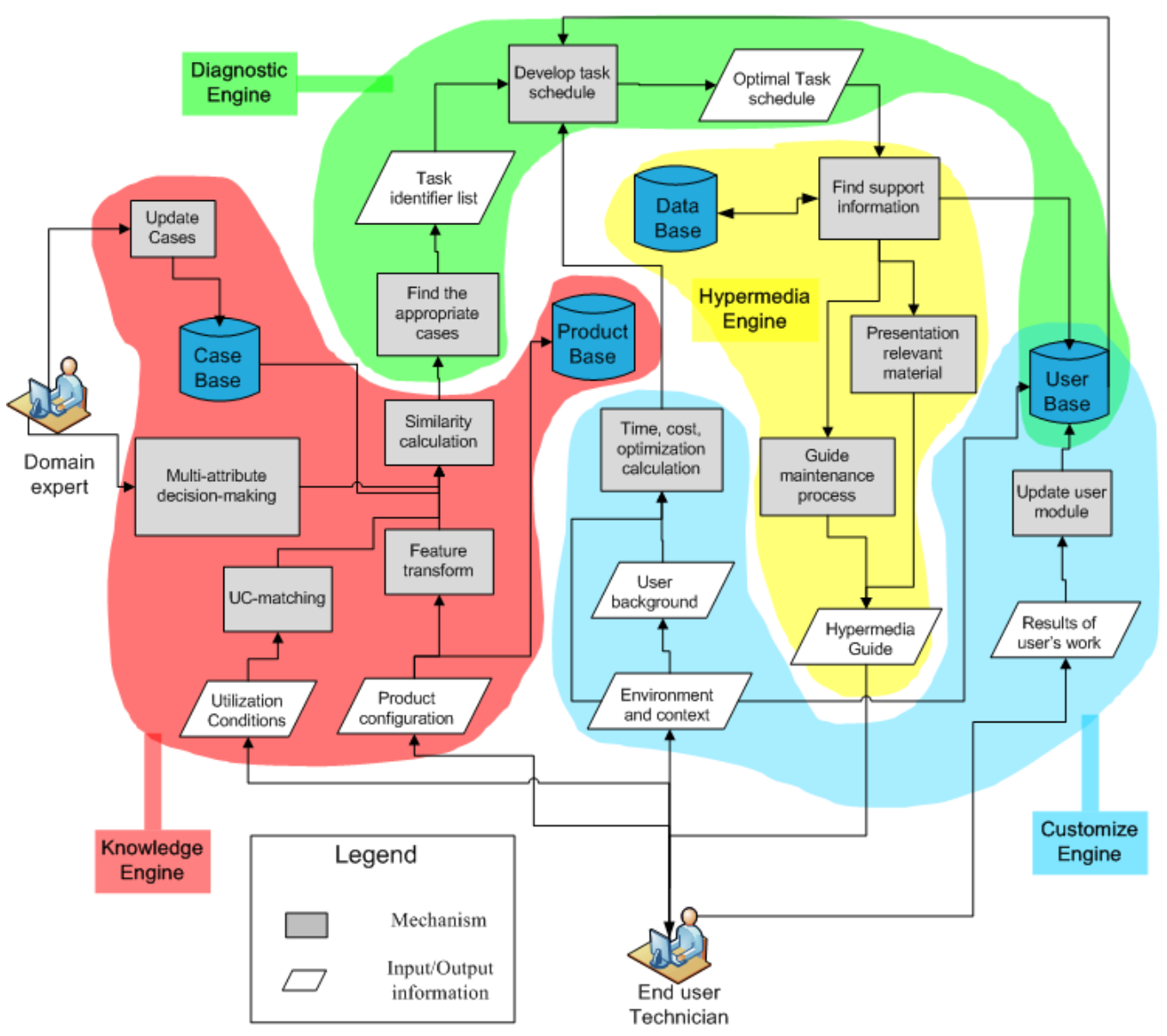

Figure 3. Framework of CMDs

2. Diagnostic Engine optimizes a maintenance schedule in order to improve the service quality and minimize the overall cost. Once the most similar case is located, the maintenance strategy is decided. Then, the maintenance schedule is calculated by the diagnostic engine according to time, availability of tools and materials, etc.

3. Customizing Engine recommends an appropriate information level and form according to the technician's level of skill and experience. A set of information collected from clients (such as skill level, experience, knowledge, education, age, and favorites) is used to determine the presentation form (such as level of abstraction or granularity, level of explanation, and media type). User profile should be updated based on client satisfaction.

4. Finally, an identifier of the selected task is passed to the Hypermedia Engine, which retrieves task-related support information from the database. The user interacts with the Hypermedia Engine through a standard Web browser. The Hypermedia Engine dynamically presents the relevant material for each performed subtask according to the user's feedback for the next cycle of work.

\section{Exemplary Case}

The case of demo in principle, a transportation enterprise, spanned the operational life of trucks from July to December, 2007, in Canada. Data were collected about 4 heavy-duty trucks with a total of 500 drive hours. The data included all significant maintenance events -- namely, installa- 
tions, failures, preventive and corrective maintenance, and modifications. The data supplied by the transportation enterprise focused on the number of maintenance works carried out on the subsystem level.

\section{Knowledge Preparation and Re-Use}

Knowledge is the basic building material for knowledge-based decision support system. In order to use the previous knowledge, historical data and previous experience are collected and mined into useful knowledge. Here, groups of information related to product, user, environment, and maintenance were collected from various resources, such as records of human resource, equipment records, maintenance records, and the federal government agency.

\section{Representation of cases}

Each truck was discomposed into eleven subsystems: pneumatic, engine, structure, electric circuit, braking circuit, and so on. Due to space limitations, only the top seven subsystems were listed. "The way of operating" can be measured by other four factors, therefore twelve input factors were used in this study, as detailed in Figure 4.

In order to inspect the performance of each truck more effectively and correctly, data were collected each week. Thus, there were almost twenty cases (maintenance events) for each truck and eighty cases in total. The dataset was then randomly divided into a calibration dataset $(75 \%)$ and a test dataset $(25 \%)$.

\begin{tabular}{|c|c|c|c|c|c|c|c|}
\hline. $\mathrm{D}$ & \multicolumn{7}{|l|}{ BI0 } \\
\hline. $\mathrm{UC}$ & \multicolumn{7}{|c|}{ UC_001 } \\
\hline User & \multicolumn{7}{|c|}{ User_002 } \\
\hline \multirow{3}{*}{ Environment } & \multicolumn{3}{|c|}{ Temperature $\left({ }^{\circ} \mathrm{C}\right)$} & \multicolumn{4}{|l|}{17} \\
\hline & \multicolumn{3}{|c|}{ Precipitation (mm) } & \multicolumn{4}{|l|}{100} \\
\hline & \multicolumn{3}{|l|}{ Dust } & \multicolumn{4}{|l|}{ Fair } \\
\hline \multirow{3}{*}{$\begin{array}{l}\text { Product } \\
\text { usage }\end{array}$} & \multicolumn{3}{|c|}{ Frequency of use (km/week) } & \multicolumn{4}{|l|}{2076} \\
\hline & \multicolumn{3}{|c|}{ Terrain } & \multicolumn{4}{|l|}{ Poor } \\
\hline & \multicolumn{3}{|c|}{ Load } & \multicolumn{4}{|c|}{ Very high } \\
\hline \multirow{4}{*}{ User profile } & \multicolumn{3}{|c|}{ Experience of operate } & \multicolumn{4}{|l|}{ Low } \\
\hline & \multicolumn{3}{|c|}{ Skill level } & \multicolumn{4}{|l|}{ Low } \\
\hline & \multirow{2}{*}{\multicolumn{3}{|c|}{ The way of operate }} & \begin{tabular}{|l|} 
Average \\
speed \\
(km/h) \\
\end{tabular} & $\begin{array}{l}\text { Average } \\
\text { RPM }\end{array}$ & $\begin{array}{l}\text { Cons, } \\
\text { Stop (L) }\end{array}$ & $\begin{array}{l}\text { Cons, } \\
\text { (L/100KM) }\end{array}$ \\
\hline & & & & 48 & 1689 & 6,2 & 125.1 \\
\hline \multirow[t]{2}{*}{ Order/Rank } & N1 & N2 & N3 & N4 & N5 & N6 & N7 \\
\hline & FS 2 & FS 10 & FS 4,9,11 & $\begin{array}{l}\text { FS } \\
4,9,11\end{array}$ & FS $4,9,11$ & FS 5 & FS 3 \\
\hline $\begin{array}{l}\text { Confidential } \\
\text { code }\end{array}$ & $22 \%$ & $18 \%$ & $12 \%$ & $12 \%$ & $12 \%$ & $11 \%$ & $5 \%$ \\
\hline
\end{tabular}

Figure 4. Example case from automobile domain

\section{Capturing new case}

New cases were captured through the interface showed in Figure 5. The user was asked to express his/her Utilization Conditions (on the scale of 1-10) on some qualitative features about the automobile. As can be seen in this figure, some descriptions were also provided to assist the user in indicating his/her Utilization Conditions. For example, it is different for the user to identify the exact temperature of the environment, but he/she can describe it in subjective terms such as "hot", "cold" or "freezing". In addition, normalization was carried out on the qualitative features given by each individual in the questionnaire. 


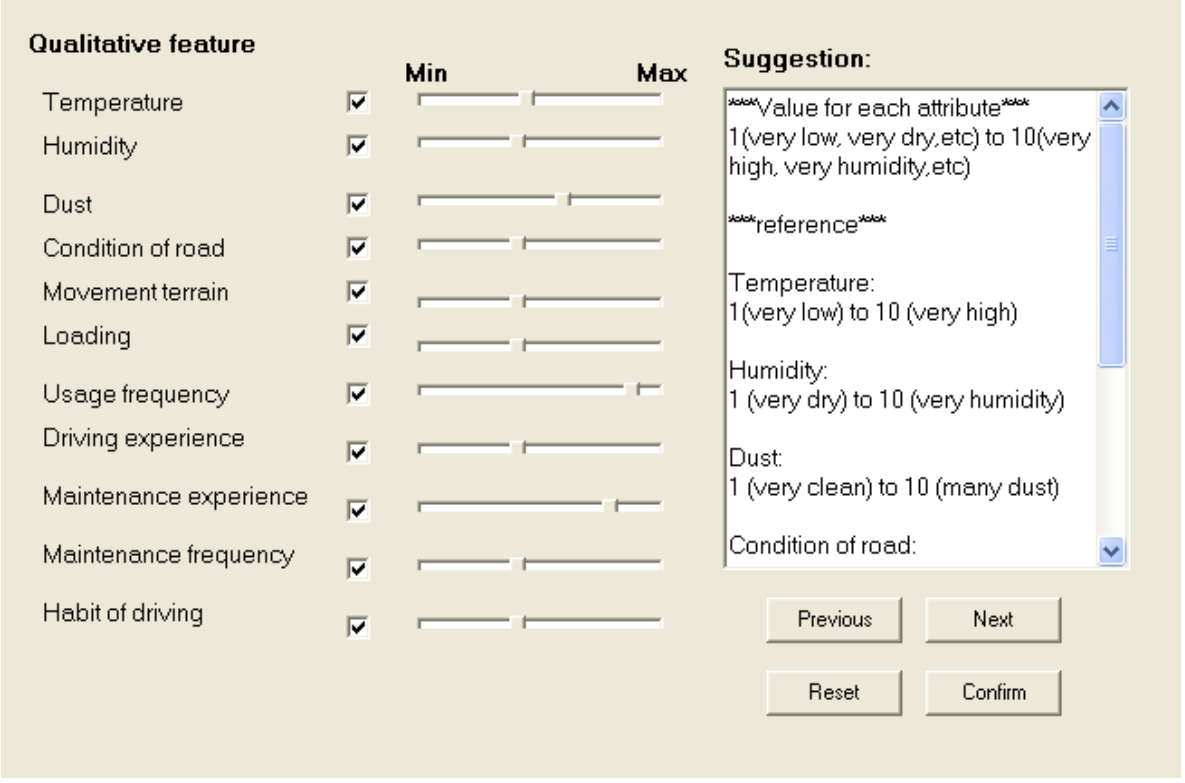

Figure 5. The questionnaire presented by the interface module

\section{Similarity calculation and case matching}

After relevant information was gathered from the user, these qualitative features were then transformed into a point in three-dimensional space. Exiting cases were clustered into six groups, and the center of each group was represented the same way - as a point in the space. Distances between the current case and group centers were calculated using the weighted arithmetic average (WA) method. Then, the current case was assigned to a group to which it had the shortest distance. It should be noted that a threshold $\mathrm{T}$ was predefined by experts; that is to say, the smallest distance should not be above the threshold. There are two possible scenarios: (1) if the smallest distance is less than or equal to $\mathrm{T}$, the current case belongs to the corresponding existing group, and (2) otherwise, it becomes the center of a new group.

For the first condition, maintenance activities of other cases in the same group can be recommended, as they have similar Utilization Conditions. Maintenance activities can be ranked based on their probabilities of occurrence. Then, what seems "the most ideal result" can be recommended to the technician. After the most similar case (or cases) is found, the differences between the past case and the current case can be analyzed. Then, the system determines which elements can be used directly and which should be modified, based on the task taxonomy and the effect of Utilization Conditions on implementation time of tasks. Furthermore, appropriate improvements may be carried out with the help of expert experience and knowledge, as explained in the next section.

For the second condition, the current case does not belong to any existing group but instead forms a new group of its own. It is difficult to recommend by existing cases, and the intervention of domain experts are needed.

\section{Customized task recommendation}

In order to extract knowledge about the Utilization Conditions of the mechanical product, the interface module communicates with human experts through an interactive process, by which the experts can enter their personal knowledge into the system. Under the circumstances designed below, the system can collect knowledge from experts to give more objective suggestions (Jaime, Gardoni, \& Frank, 2006; Gardoni, 2005). 
Figure 6 shows the screens where the expert chooses the affected maintenance activities and adjusts their implementation time. Activities are classed into seven groups according to the effect of Utilization Conditions in the case of automobile.
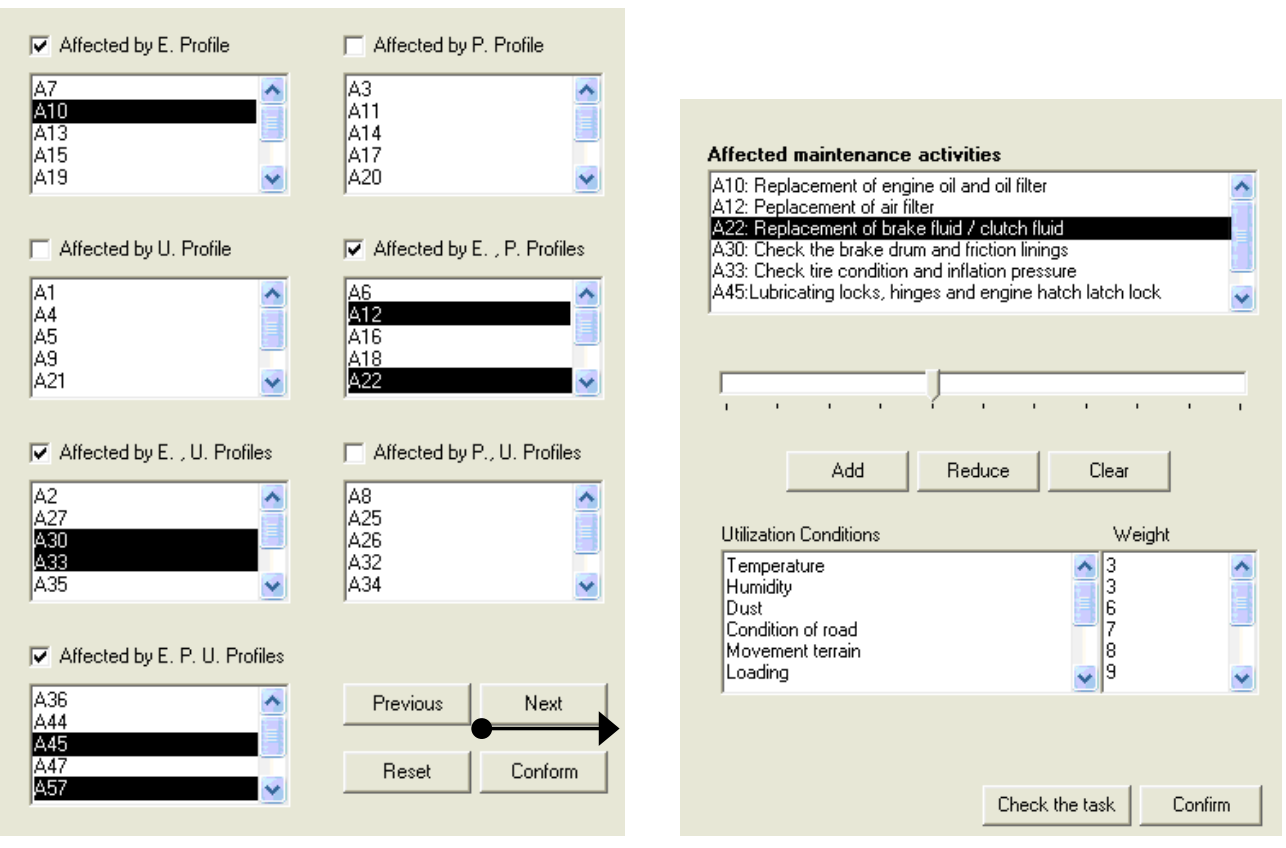

Figure 6. The interface for an expert to choose affected activities

In the screen shown in the left section of Figure 6, the expert analyzes which activities' timing need to be modified in comparison with the standard case, and then presses the 'Confirm' button to choose them. After that, the expert can further adjust the implementation time of the chosen activity or activities, as shown in the right section of Figure 6. In this part of interface, when the expert chooses an affected task (e.g., 'A22') and presses the 'Check the task' button, the related information elements (such as required condition, required person, maintenance procedures, required skill levels, and the estimated time) for implementing the task are shown on a new page. The expert can select one affected task and move the bar in the middle to adjust the implementation time of this task. The expert can then press the 'Add' button to schedule the implementation of this task. The lower part of this interface shows the list of Utilization Conditions and weights previously assigned. As mentioned earlier, different experts may have their own settings, and here the average is used to define each performance value.

\section{Results and Discussion}

The user's experience and roles are captured after his/her login, and the failure rates of components are predicted by the CMD system. As a result, appropriate maintenance tasks should be assigned according to health conditions of these components. In the comparative analysis below, it is assumed that four trucks were driven by two drivers separately, one experienced (D1) and the other novice (D2). They were driven in a similar terrain, and itinerary, load and utilization frequency were almost the same. Thus, product usage profiles would be the same as well. Eight components were studied: I1 (Pneumatic circuit), I2 (Pneumatic), I3 (Transmission), I4 (Engine), I5 (Electric circuit), I6 (Braking circuit), I7 (Planetary and hubs), I8 (Structure).

Figure 7 illustrates the failure rates of components for trucks driven by the two different drivers, experienced 7(a) and novice 7(b). In 7(a), the failure rate of each component does not exceed $40 \%$, while in 7 (b) the rates of three components (I2, I4, and I5) exceed $40 \%$. The way of driving 
sometimes can affect the lifetime of the pneumatic, such as harsh braking, fierce acceleration, and fast cornering. It is noteworthy that there are fewer failure components in 7(b), with components I1, I3, and I8 appearing only in 7(a). This is different from what we expected -- that drivers with low speed would have more failures than those with high speed in the worse conditions, although the latter are more oil saving.

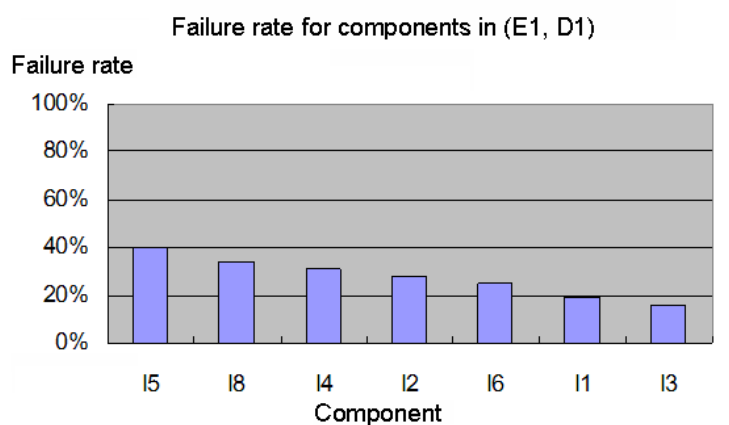

(a)

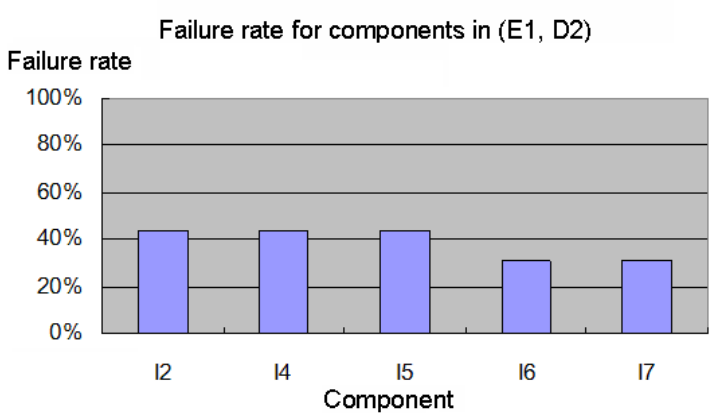

(b)

Figure 7. Failure rates of components for (a) experienced and

(b) novice drivers in the same environment

Figure 8 shows failure rates of components under two different environments (E1 and E2). Compared with $8(\mathrm{~b})$, there are two additional components in $8(\mathrm{a})$ : I4 and I1. It is noteworthy that the failure rate of I2 is significantly higher in environment E2. Two typical seasons are noticed, one being high humidity with high temperature, and the other high humidity with low temperature, since the data were gathered from July to December in Canada. It is not difficult to understand that there are more failed components in the first environment (high humidity with high temperature) than in the second. From the interviews with experts, we learned that there were potholes caused by the presence of water and high humidity. The uneven ground with heavy load caused the excessive pneumatic wear.

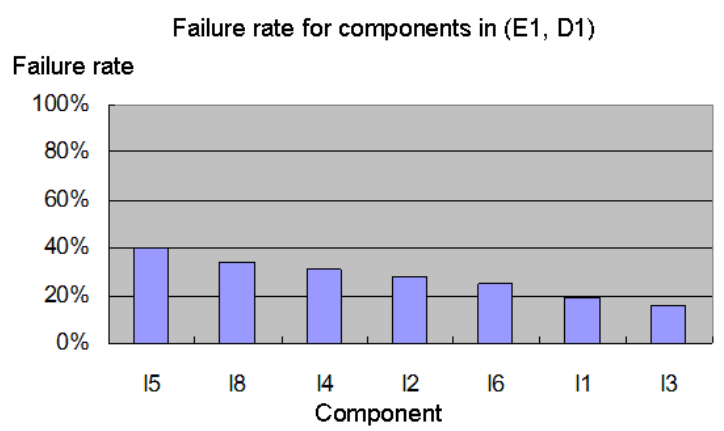

(a)

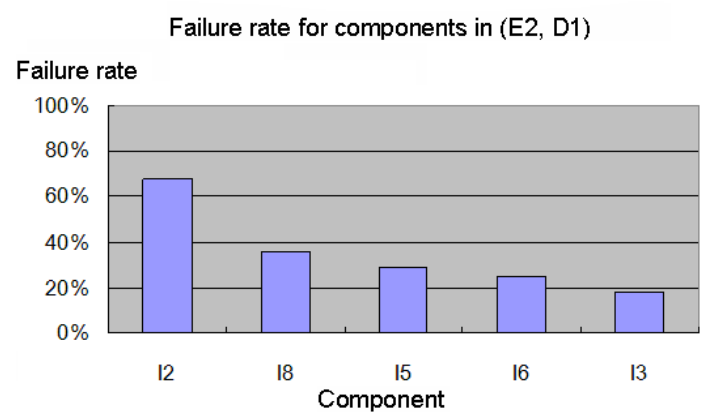

(b)

Figure 8. Failure rates of components by the experienced driver (D1) in two different environments (E1 and E2)

\section{Interface of CMDs}

The hypermedia interface of CMDs consists of two main windows: the outline frame (left section), and the content presentation frame (right section), as shown in Figure 9. Each frame can present several types of information. The user can select the desired type of information using the named tabs at the top of the content frame. 


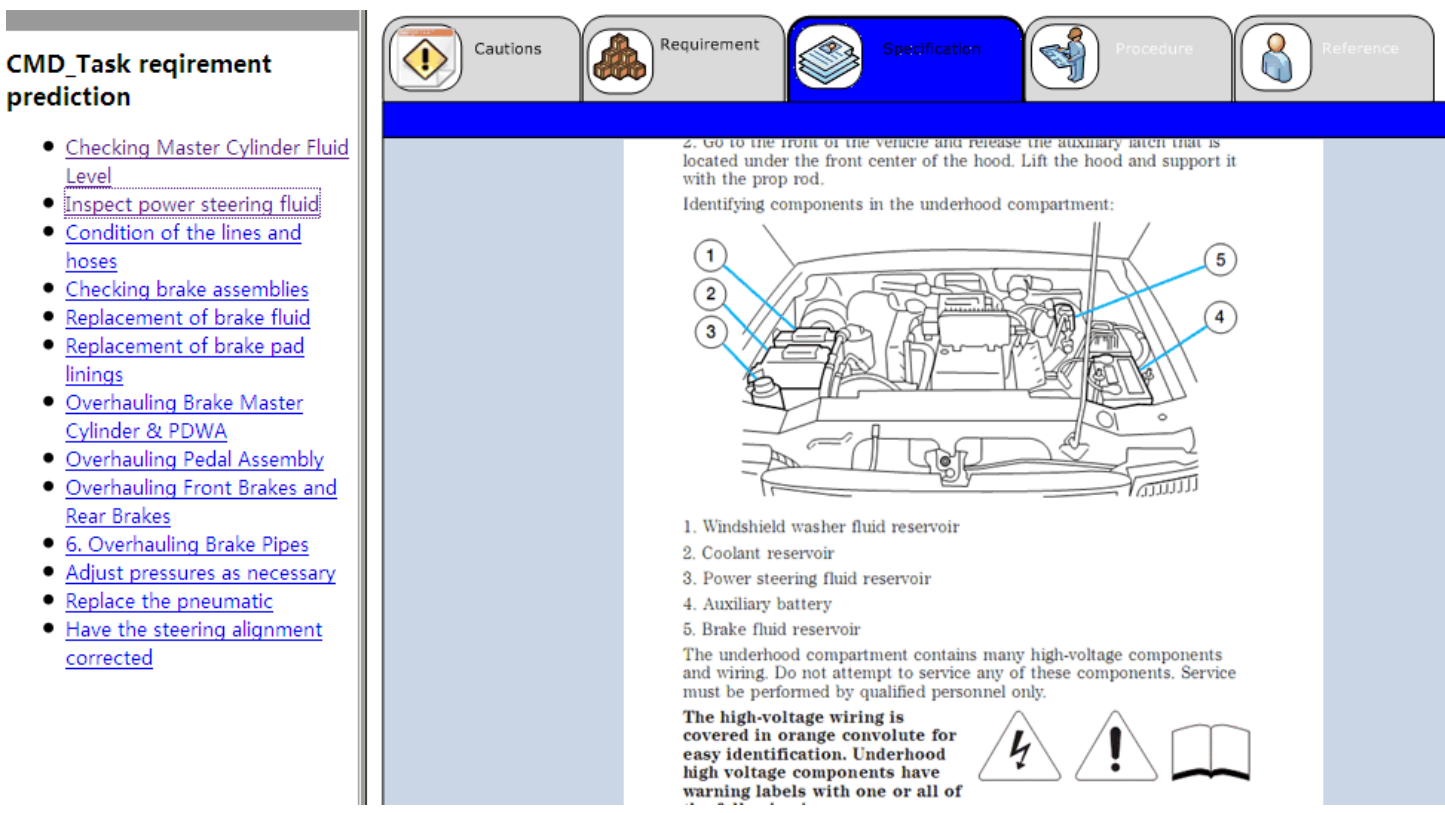

Figure 9. The task predicting interface with outline and content presentation frames

The main function of the outline frame is to provide a customized maintenance program board of the task being performed in line with the utilization conditions of the current product. It helps the user to decide what task should be performed without spending too much time.

The content presentation frame is to display relevant support information for the task selected in the outline frame. Several navigation "tabs" are provided to organize the support information into categories by types and present each type in a separate window. As explained earlier, information objects are organized in a task-oriented fashion; all elements of support information are assembled together to further explain the task. An abundance of content is presented to the user, such as requirements needed to perform the task, specifications of the components involved, and procedures of completing the task. Furthermore, warnings and cautions are presented to inexperienced users, which help them avoid being hurt accidentally. References are provided for users to find supplementary information to increase their understanding.

\section{Conclusions}

This paper presents a knowledge integration approach for elaborating Customized Maintenance Documents and demonstrates how to customize the document in line with different maintenance strategies triggered by variety of utilization conditions and constraints, such as available tools and materials, technician experience and education, and environment. In order to make the CMD easy to update and adapt according to various utilization conditions and constraints, a three-layer knowledge modeling is proposed as the information organization mechanism. A system of four collaborative engines was implemented to integrate expert knowledge and historical information so that the user and technician could make the right decision during maintenance process. Specifically, the knowledge engine retrieves the most similar case. Once this case is obtained, the maintenance schedule is calculated by the diagnostic engine. Then, the customizing engine recommends an appropriate information level and form according to the skill and experience level of the technician. Finally, the selected task and the relevant information are displayed by the hypermedia engine, aiming to provide customized and task-oriented maintenance support. As demo in principle, empirical data were collected from an exemplary case of truck transportation and analyzed to illustrate the effectiveness of the approach. 
Huang, Wang, Gardoni, \& Amadou

Further research is needed in this area to study modeling and simulation for maintenance programme planning. Another important area for future research is to extend the framework to skill training of inexperienced technicians.

\section{References}

Batanov, D., Nagarur, N., \& Nitikhunkasem, P. (1993). EXPERT-MM: A knowledge-based system for maintenance management. Artificial Intelligence in Engineering, 8(4), 283-291.

Bertolini, M., \& Bevilacqua, M. (2006). A combined goal programming - AHP approach to maintenance selection problem. Reliability Engineering and System Safety, 91(7), 839-848.

Brodie, D., Gupta, A., \& Shi, W. S. (2005). Accelerating dynamic Web content delivery using keywordbased fragment detection. Journal of Web Engineering, 4(1), 79-99.

Cooper, D. W. (1999). Adaptive diagnostics and personalized technical support (ADAPTS). Proceedings of IEEE Aerospace Conference, vol. 3, 139-149.

Gardoni, M. (2005). Concurrent engineering in research projects to support information content management. Concurrent Engineering: Research and Applications (CERA), 13(2), 135-144.

Huang, Y., Gardoni, M., \& Coulibaly, A. (2009). A recommendation system designed for adaptive maintenance support. International Conference on Engineering Design ICED'09, Stanford University, Stanford, CA, USA, 24 - 27 August 2009.

Jaime, A., Gardoni, M., \& Frank, C. (2006). Communications tools in research projects to support semi and non structured information. Journal of Systemics, Cybernetics and Informatics, 3(3), 85-93.

Lee, J., Ghaffari, M., \& Elmellgy, S. (2011). Self-maintenance and engineering immune systems: Towards smarter machines and manufacturing systems. Annual Reviews in Control, 35(1), 111-122.

Mathur, A., Ghoshal, S., Haste, D., Domagala, C., Shrestha, R., Malepati, V., \& Pattipati, K. (2000). An integrated support system for rotorcraft health management and maintenance. IEEE Aerospace Conference Proceedings, 6, 18-25.

Pham, D. T., Dimov, S. S., \& Huneiti, A. M. (2003). Semantic data model for product support systems. Industrial Informatics, INDIN 2003. Proceedings, 279-285.

Pham, D. T., Dimov, S. S., \& Soroka, A. J. (2001). Knowledge acquisition for intelligent product manuals. Proceedings of the institution of Mechanical Engineers, Part B: Journal of Engineering Manufacture, 215 (1), 97-103.

Pham, D. T., \& Setchi, R. M. (2001). Integrating case-based reasoning and hypermedia documentation for intelligent product support. 17th National Conference on Manufacturing Research (NCMR), 111-116.

Setchi, R. M., Lagos, N., \& Huneiti, A. (2007). Semantic modeling of product manuals. Knowledge-Based Intelligent Information and Engineering Systems, 11th International Conference, KES 2007, XVII Italian, 1162-1169.

Setchi, R. M., Pham, D. T., \& Dimov, S. S. (2006). A methodology for developing intelligent product manuals. Engineering Applications of Artificial Intelligence, 19(6), 657-669.

Van Houten, F. J. A. M., \& Kimura, F. (2000). The virtual maintenance system: A computer-based support tool for robust design, product monitoring, fault diagnosis and maintenance planning. Annals of the ClRP-Manufacturing Technology, 49(1), 91-94.

Xia, T., Xi, L, Lee, J., \& Zhou, X. (2011). Optimal CBPM policy considering maintenance effects and environmental condition. International Journal of Advanced Manufacturing Technology, 56(9-12), 11811193. 


\section{Biographies}

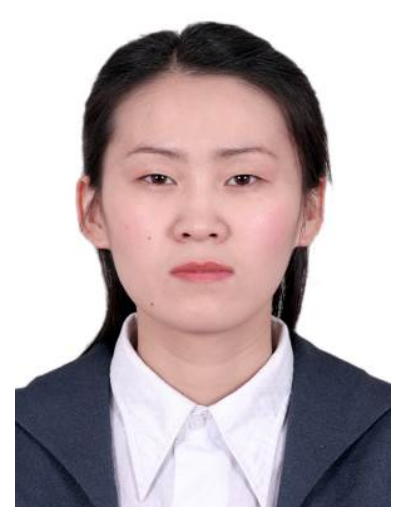

Y. Huang, currently a Lecturer in Taizhou University, China in 2013. She obtained her PhD degree from University of Strasbourg, France, in 2012, specializing in Information and Knowledge Management for Design and Manufacturing. She is interested in elaborating the maintenance document for complex mechanical products with different utilization conditions.

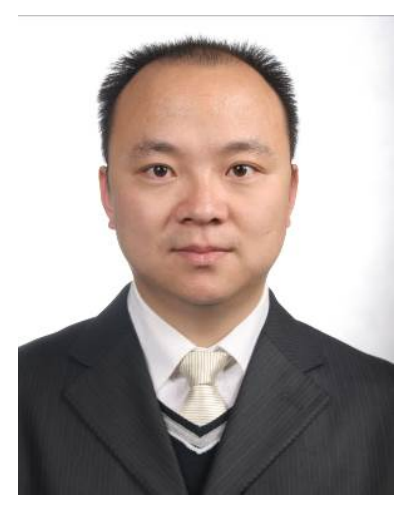

X. J. Wang, Assistant Professor of Mechanical Engineering in Qiannan Nornal Colledge for Nationalities, China. He obtained his PhD degree from INSA of Lyon on impact location using pizoelectic sensors. He is interested in the field of machine maintenance such as: Structural Health Monitoring and Condition-based maintenance.

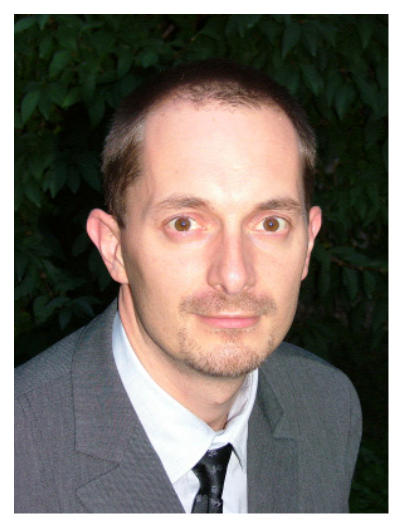

M. Gardoni, Professor and Director of the innovation management program at ÉTS (Québec - Canada) and professor at INSA de Strasbourg. He was professor at INP Grenoble (France) and CoDirector of the "French-Chinese PLM Centre for Innovation" in Tsinghua University, Beijing, China. He is engineer in industrial engineering and has done his $\mathrm{PhD}$ in EADS (European Aeronautic Defence and Space Company). His research interests include methodologies of creativity-innovation and knowledge management. 


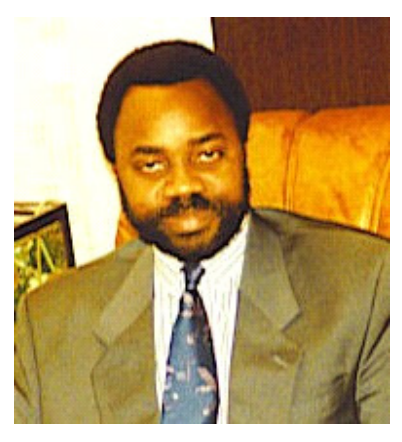

Amadou Coulibaly, Associate Professor at Graduate School of National Institute of Applied Sciences of Strasbourg (INSA de Strasbourg France) in the Engineering Design Laboratory (LGECO). He is a Mechanical Engineer from Ecole Polytechnique de Thiès (Senegal) and $\mathrm{PhD}$ in Mechanical Engineering from Louis Pasteur University of Strasbourg (France). His research interests concern Semantic Design, Semantic CAD and Product Behavioural Performance Engineering. His current research industrial applications are focused on reliability, maintainability and recycle ability assessment in innovative mechatronic systems. 